\title{
Noncontact speckle contrast diffuse correlation tomography of blood flow distributions in tissues with arbitrary geometries
}

Siavash Mazdeyasna

Chong Huang

Mingjun Zhao

Nneamaka B. Agochukwu

Ahmed A. Bahrani

Lesley Wong

Guoqiang Yu 


\title{
Noncontact speckle contrast diffuse correlation tomography of blood flow distributions in tissues with arbitrary geometries
}

\author{
Siavash Mazdeyasna, ${ }^{a}$ Chong Huang, ${ }^{a}$ Mingjun Zhao, ${ }^{a}$ Nneamaka B. Agochukwu, ${ }^{b}$ Ahmed A. Bahrani, ${ }^{a, c}$ \\ Lesley Wong, ${ }^{b}$ and Guoqiang $\mathbf{Y u}^{\mathrm{a}, *}$ \\ aUniversity of Kentucky, F. Joseph Halcomb III, M.D. Department of Biomedical Engineering, Lexington, Kentucky, United States \\ bUniversity of Kentucky, Division of Plastic Surgery, Lexington, Kentucky, United States \\ 'University of Baghdad, Al-Khwarizmi College of Engineering, Biomedical Engineering Department, Aljadriya, Baghdad, Iraq
}

\begin{abstract}
A noncontact electron multiplying charge-coupled-device (EMCCD)-based speckle contrast diffuse correlation tomography (scDCT) technology has been recently developed in our laboratory, allowing for noninvasive three-dimensional measurement of tissue blood flow distributions. One major remaining constraint in the scDCT is the assumption of a semi-infinite tissue volume with a flat surface, which affects the image reconstruction accuracy for tissues with irregular geometries. An advanced photometric stereo technique (PST) was integrated into the scDCT system to obtain the surface geometry in real time for image reconstruction. Computer simulations demonstrated that a priori knowledge of tissue surface geometry is crucial for precisely reconstructing the anomaly with blood flow contrast. Importantly, the innovative integration design with one single-EMCCD camera for both PST and SCDCT data collection obviates the need for offline alignment of sources and detectors on the tissue boundary. The in vivo imaging capability of the updated scDCT is demonstrated by imaging dynamic changes in forearm blood flow distribution during a cuff-occlusion procedure. The feasibility and safety in clinical use are evidenced by intraoperative imaging of mastectomy skin flaps and comparison with fluorescence angiography. () 2018 Society of Photo-Optical Instrumentation Engineers (SPIE) [DOI: 10.1117/1.JBO.23.9.096005]

Keywords: diffuse optical imaging; blood flow distribution; tissue surface geometry; mastectomy skin flap necrosis.

Paper 180349R received Jun. 13, 2018; accepted for publication Sep. 4, 2018; published online Sep. 24, 2018.
\end{abstract}

\section{Introduction}

Blood flow at the microcirculatory level is responsible for the delivery of oxygen and nutrients to tissue and the removal of metabolic by-products. Measurement of tissue blood flow provides crucial information for both the diagnosis and therapeutic monitoring of many vascular/cellular diseases including stroke, head trauma, and peripheral arterial disease. ${ }^{1}$ These diseases are usually associated with regional tissue ischemia and hypoxia; thus imaging of tissue blood flow distribution may have a role in the further delineation of these disease states. Threedimensional (3-D) imaging ability is of importance for the monitoring of diseases affecting deep tissue volumes. Noncontact imaging devices are needed for investigating the blood flow status of vulnerable tissues such as burned tissues, wounds, and reconstructive tissue flaps.

There are currently very few noninvasive (or minimal-invasive) imaging tools available for blood flow assessments including nuclear medicine, x-ray imaging, magnetic resonance imaging (MRI), ultrasound techniques, and optical methods. ${ }^{2}$ In contrast to these large imaging modalities (i.e., nuclear medicine, x-ray imaging, and MRI) and the ultrasound measurement of blood flow velocity in large vessels, optical imaging techniques based on dynamic light scattering offer noninvasive, fast, continuous, inexpensive, and portable means for blood flow measurements in tissue microvasculature. Examples of these optical imaging techniques include laser speckle contrast imaging (LSCI) $)^{3,4}$ and near-infrared (NIR) diffuse correlation spectroscopy/tomography (DCS/DCT). ${ }^{5-12}$ Operating in a noncontact manner, LSCI uses wide-field coherent illumination and charge-coupled-device (CCD) to detect spatial or temporal fluctuations of laser speckle contrasts, which enables rapid high-resolution two-dimensional (2-D) imaging of blood flow distribution on the superficial tissue with a depth $<1 \mathrm{~mm}$. $3,4,13,14$ In contrast, DCS/DCT uses point-source coherent illuminations and discrete avalanche photodiodes (APDs) to detect temporal fluctuations of laser speckle contrasts for spectroscopic/tomographic (3-D) measurements of blood flow variations in deep tissue volumes (up to $\sim 15-\mathrm{mm}$ depth)..$^{5-12}$

Traditional DCS/DCT systems use fiber-optic probes in contact with the tissue surface for blood flow measurements. We have innovatively developed a noncontact DCS/DCT (ncDCS/ncDCT) system for the measurement/imaging of blood flow distributions in deep tissue volumes. ${ }^{10,15}$ The advantage of noncontact measurements using our unique lens-focusing technique makes the ncDCS/ncDCT particularly suitable for monitoring incisions and open tissues with no risk for contact infection. Also, by not requiring an intravenous injection like other methods (e.g., fluorescence angiography), the risk of an allergic reaction is avoided. Although effective, ncDCS/ncDCT employs a limited number of expensive long-coherence lasers and APDs (SPCM-AQR-12, PerkinElmer) for blood flow detection, leading to poor temporal-spatial resolution and high cost, not to mention the large dimensions of the instrument. 
To overcome these limitations, we have recently developed a noncontact CCD-based speckle contrast diffuse correlation tomography (scDCT) system, ${ }^{16-18}$ which combines the benefits of fast and high-resolution CCD detection with the unique finite-element-method (FEM)-based DCT reconstruction technique, ${ }^{11,12}$ allowing for 3-D imaging of blood flow distributions in relatively deep tissues (up to $\sim 10 \mathrm{~mm}$ ). What distinguishes the $C C D$ detection in $\mathrm{scDCT}^{16-18}$ from the APD detection in DCS/DCT ${ }^{10-12,15,19,20}$ is the transition from measurements of slower temporal fluctuations (hundreds of milliseconds) to faster spatial fluctuations (a few milliseconds) of laser speckles, allowing for much higher temporal resolution. Furthermore, hundreds of 2-D-array detectors provided by the CCD camera dramatically increase the sampling density and make the scDCT device more portable and less expensive than DCS/DCT. The scDCT system has been tested for 3-D imaging of flow distributions in tissue-simulating phantoms, human forearms, and mastectomy skin flaps. ${ }^{16-18}$ Results demonstrate that scDCT enables a fast and high-density 3-D flow imaging of deep tissue volumes over an adjustable region of interest (ROI). One major remaining constraint is the assumption of a semi-infinite tissue with flat surface, which may affect the accuracy of image reconstruction for tissues with irregular geometries. In addition, the irregular tissue geometry may result in the defocusing of sources and detectors, leading to source-detector (S-D) distance errors. In our ncDCT technique, we have previously used a commercial 3-D photogrammetric scanner to obtain tissue surface geometry. ${ }^{11,12}$ Although effective, this method requires the scanning of tissue surface and the offline S-D alignment and registration on the tissue surface for image reconstruction. Both procedures are time consuming, which constrains the utility of ncDCT in the clinic. Also, coregistration of two image systems (i.e., ncDCT and photogrammetric scanner) is not trivial. ${ }^{12}$

In this study, we explored using one single-CCD camera to obtain both tissue surface geometry and boundary flow data. An advanced 3-D photometric stereo technique (PST) was adapted to obtain the surface geometry in real time. PST was originally introduced to evaluate the surface orientation from multiple images taken at the same viewpoint under different illumination directions. ${ }^{21}$ PST was recently explored as a fast and inexpensive tool for face recognition at security check points. ${ }^{22}$ We innovatively integrated the PST into our scDCT system via attaching four light-emitting diodes (LEDs) to the CCD camera used for the scDCT data collection. Four 2-D images obtained from the camera perspective with four different illuminations by the LEDs were used to reconstruct the surface geometry. A tetrahedral volume mesh based on the obtained tissue surface geometry was then generated for 3-D reconstruction of blood flow distributions using scDCT technique. ${ }^{11,12}$ To evaluate the performance of the integrated scDCT system, computer simulations were first conducted to characterize the influence of surface geometry in the reconstruction of blood flow distributions. The modified scDCT system was then tested to image forearm blood flow distribution in response to a cuff-occlusion on the upper arm. Finally, the scDCT instrument was moved to the surgical room for intraoperative imaging of mastectomy skin flaps. This paper reports the first imaging system using a single-CCD camera to obtain both tissue surface geometry and boundary blood flow distribution, which obviates the need for offline complex alignment of sources and detectors on tissue boundary, and improves the accuracy of blood flow image reconstruction.

\section{Materials and Methods}

\subsection{Noncontact scDCT System}

Figure 1 shows the schematic diagram of scDCT device. Briefly, a long coherence laser $(785 \mathrm{~nm}$, CrystaLaser) was used as a point source. A galvo mirror was used to remotely deliver coherent point light to source positions for sequencing of tomographic data acquisition. An electron multiplying charge-coupled-device (EMCCD, Cascade 1K, Photometrics) camera was used to measure spatial diffuse speckle contrasts on the tissue boundary in a selected ROI. A graphical interface (LabVIEW) was created to control the galvo mirror and send triggers to the EMCCD for acquiring images sequentially at multiple source positions.

A high-performance long-pass filter (>750 nm, \#84-761, EdmundOptics) with the optical density larger than 4.0 was used in our scDCT to minimize the influence of ambient light. The percentage transmission of visible light through this longpass filter is below $10^{-4}$. Moreover, light in the operation room was dimmed when taking both fluorescence angiography and scDCT data. A pair of polarizers (LPNIRE050-B and LPNIRE200-B, Thorlabs) was added crossing the source and detection paths to reduce the source reflection directly from the tissue surface. An achromatic lens (AC127-019-B, Thorlabs, New Jersey) was installed in the source path behind the galvo mirror to ensure the focused point source light at the tissue surface. A zoom lens (Zoom 7000, Navitar) was used to adjust the ROI. The scDCT probe was fixed on a 180-deg rotating holder for easy adjustment and alignment.

The movement of red blood cells in the measured tissue volume ("banana-shape") produced spatial fluctuations of laser speckles on the tissue surface, which were captured by the EMCCD camera with a typical exposure time of $5 \mathrm{~ms}$. For data analysis, the spatial speckle contrast $(K)$ is quantified over a selected window of $7 \times 7$ pixels on the camera by simply calculating the intensity ratio of standard deviation $(\sigma)$ to mean

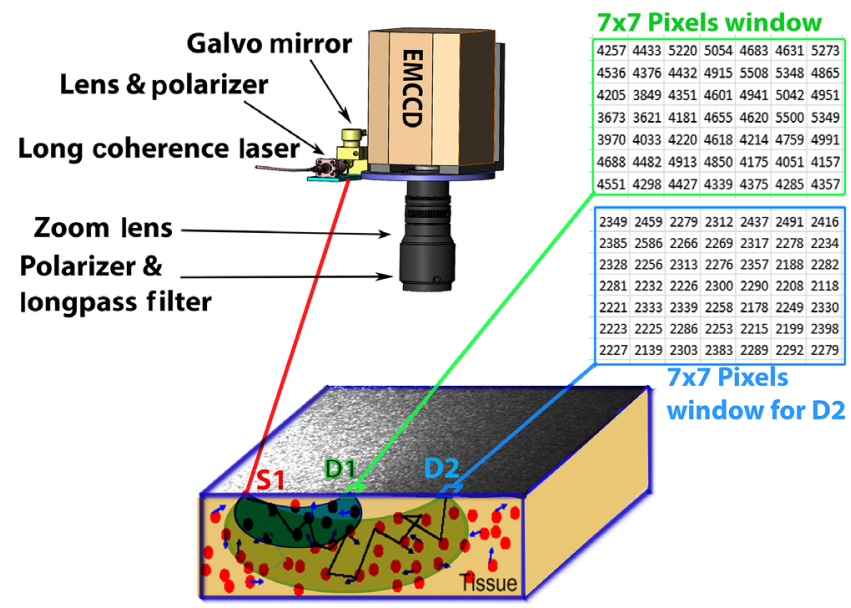

Fig. 1 The schematic of the scDCT system for 3-D imaging of tissue blood flow distribution. A galvo mirror is used to deliver the point light to different positions. Multiple pixel-windows/detectors are used to detect spatial speckle contrasts at different locations. The coherent point-source light (e.g., S1) diffuses through a "banana-shape" photon pathway inside a deep tissue volume and reaches to the detectors (e. g., D1 and D2). A long-pass filter (>750 nm) and a pair of polarizers are used to reduce the influence of ambient light and the light reflected directly from the source. A zoom lens is connected to the EMCCD camera for adjusting the size of ROI. 
$(\mu)$ over these 49 pixels; i.e., $K=\sigma / \mu$. These boundary data $K_{S-D}(r)$ calculated from multiple sources (e.g., S1, S2...) and multiple pixel-windows/detectors (e.g., D1, D2...) are input into an FEM-based program (i.e., modified NIRFAST) for 3-D reconstruction of blood flow distributions in the measured tissue volumes. ${ }^{16-18}$

\subsection{Modified Noncontact scDCT System Integrated with PST}

The scDCT system was modified to capture the surface geometry information via PST using the same EMCCD camera. Figure 2(a) shows the design for PST using four neutralwhite LEDs (SP-05-N4, Luxeon Star LEDs) installed on four holding arms, fabricated by a 3-D printer. The 2-D images were sequentially obtained by initiating LEDs to flash successively with a short camera exposure $(<1 \mathrm{~s}$ in total for flashing four LEDs). This procedure provided four separate target images, each with shading determined by a different lighting vector.

Figure 2(b) shows the PST pipeline for reconstructing the breast surface geometry in a patient undergoing a mastectomy. The surface normal map (i.e., the direction of the line extending to the centroid) is calculated using the following equation: $:^{22,23}$

$$
\left[I_{1}, I_{2}, I_{3}, I_{4}\right]^{\mathrm{T}}=\lambda N \cdot\left[L_{1}, L_{2}, L_{3}, L_{4}\right]^{\mathrm{T}},
$$

where $L_{n}$ is the known location for $n$ 'th light source ( $n=1,2,3,4)$, i.e., the coordination of each LED. Here, $I_{n}$ is the intensity of image pixels corresponding to that LED location, and $\lambda$ is the albedo at the location and $N$ is the normal surface unit. The height map is assessed using the gradient of the normal surface for the adjacent pixels. Finally, a 3-D surface geometry is created based on the height map in the selected field of view (FOV).

\subsection{Data Acquisition and Image Reconstruction}

Figure 3 shows the flowchart for data sequencing and flow image reconstruction. An FOV was first selected via adjusting the zoom lens of the EMCCD camera to cover the desired ROI. A user-friendly LabVIEW program (National Instruments) was designed to sequentially obtain four 2-D images with different LED illuminations for PST. The software then controlled the galvo mirror and sent triggers to the EMCCD for acquiring

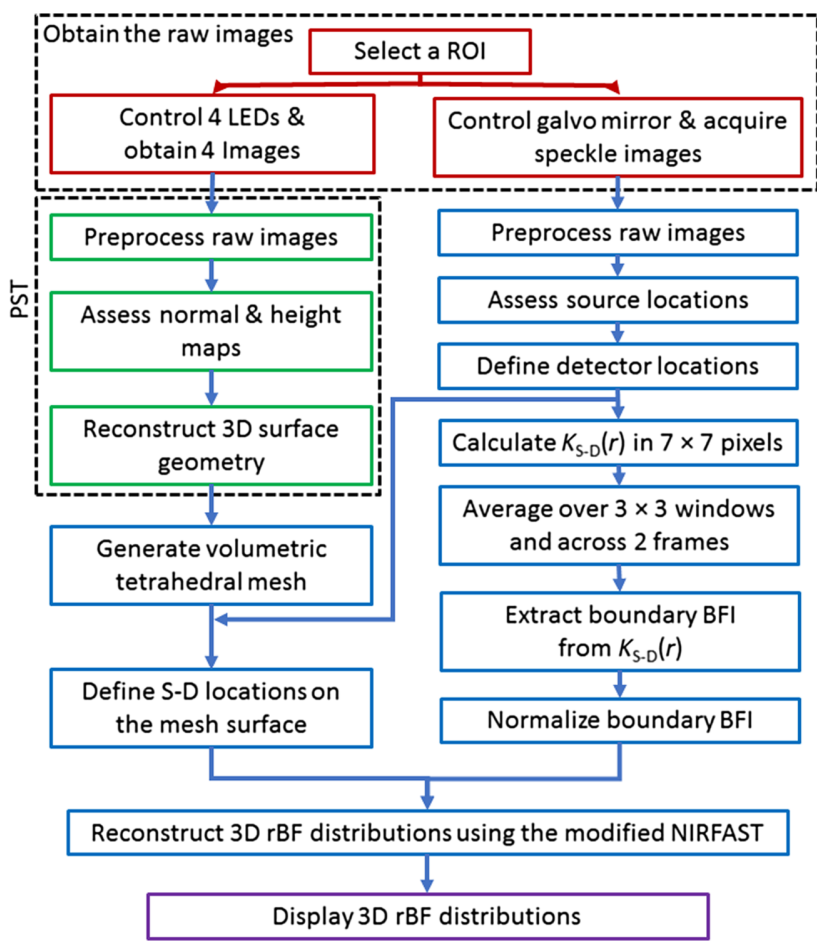

Fig. 3 The flowchart for data sequencing, processing, and flow image reconstruction using the upgraded ScDCT. The boxes with different colors represent different functions: red, ScDCT data acquisition using LabView; green, tissue surface geometry reconstruction using PST; blue, flow image reconstruction using a customized MATLAB program; and purple, result illustration using ParaView.

scDCT images sequentially at multiple source positions. These implementations are shown in the red boxes of Fig. 3.

For PST data processing, the four raw images taken with different LED illuminations were resized from $1004 \times 1002$ pixels to $100 \times 100$ pixels via a bicubic interpolation (weighted average of pixels in the nearest $4 \times 4$ neighborhood) as preprocessing to reduce the time for PST analysis from several minutes to $<2 \mathrm{~s}$. The normal map was then determined, which is the direction of the line extending to the centroid. The 3-D surface geometry was reconstructed using the normal map. These implementations are displayed in the green boxes of Fig. 3. The surface geometry reconstructed by the PST was used to generate a tetrahedral mesh.
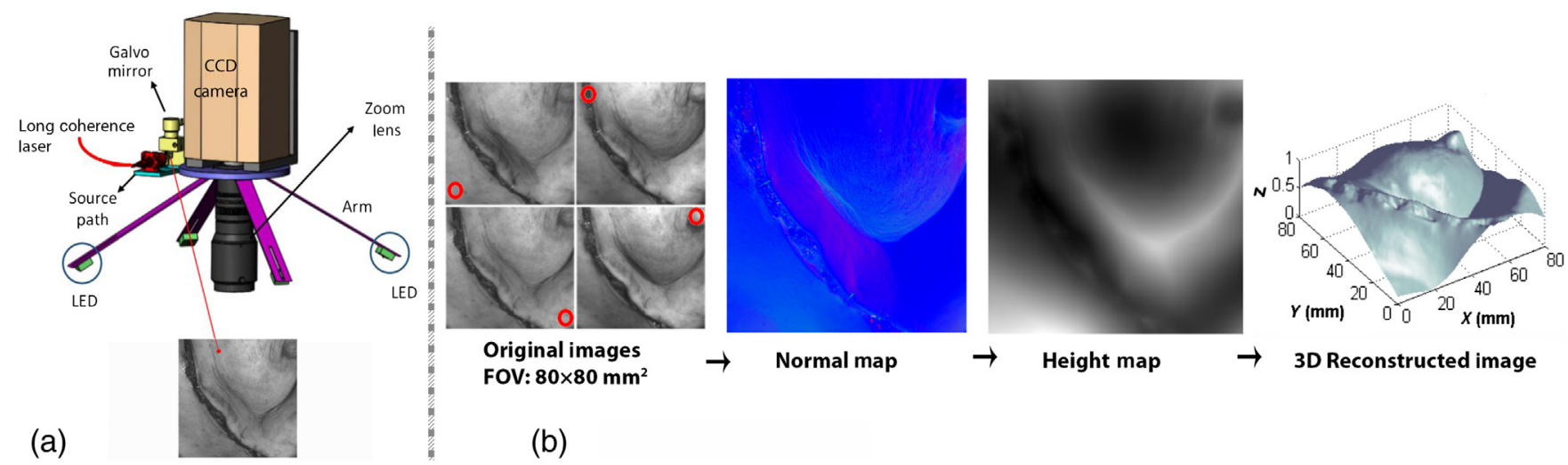

(b)

Fig. 2 The upgraded SCDCT system that integrates an advanced PST into the scDCT prototype. (a) Imaging setup with the upgraded scDCT system and (b) PST pipeline for reconstructing the tissue surface geometry. The illumination directions are indicated with red circles on original images. 
For scDCT data processing, smear correction algorithm was applied on raw images as a preprocessing for the EMCCD camera. $^{18}$ For FEM-based image reconstructions, source and detector positions must be projected on the mesh surface. In our previous study, point sources were assumed to be evenly distributed on the mesh surface, ${ }^{16}$ which might not be true if the target tissue had an irregular geometry. To precisely assess the location of each source, the intensity image with each source illumination was first converted to a simple indexed image. This was done by scaling the dynamic range and then rounding an intensity image to generate an equivalent indexed image (e.g., 16-bit integer to an indexed image with 16 indices). A morphological filter was then applied to the indexed image for removing thin protrusions and isolated areas to reduce artifacts. ${ }^{24}$ The central position of the source was finally determined by extracting pixels with regional maximum intensities on the indexed image. This process was performed on all raw images to determine all source locations. Detectors were then distributed evenly between the sources to cover the selected ROI.

For flow image reconstruction, boundary $K_{\mathrm{S}-\mathrm{D}}(r)$ at the effective S-D pairs with S-D distances ranging from 7 to $19 \mathrm{~mm}$ were calculated. ${ }^{16,18}$ Dark and shot noises were then removed when calculating the boundary speckle contrasts. ${ }^{18,25,26}$ To improve the signal-to-noise ratio (SNR), the speckle contrasts were averaged over $3 \times 3$ detector windows and across two frames. Boundary $\mathrm{BFI}(r)$ were then extracted by iteratively minimizing the difference between the measured and theoretical $K_{\mathrm{S}-\mathrm{D}}(r)$. Finally, the normalized boundary $\operatorname{BFI}(r)$ data (normalized to baseline before cuff-occlusion or normalized to the mean BFI value for mastectomy imaging) along with the tetrahedral mesh were inserted into the modified NIRFAST program, developed previously for expedient FEM-based scDCT tomographic reconstructions. ${ }^{11}$ The above processing sequences are shown in the blue boxes of Fig. 3. All data processing shown in the blue and green boxes of Fig. 3 were performed by a customized MATLAB program. The reconstructed flow images
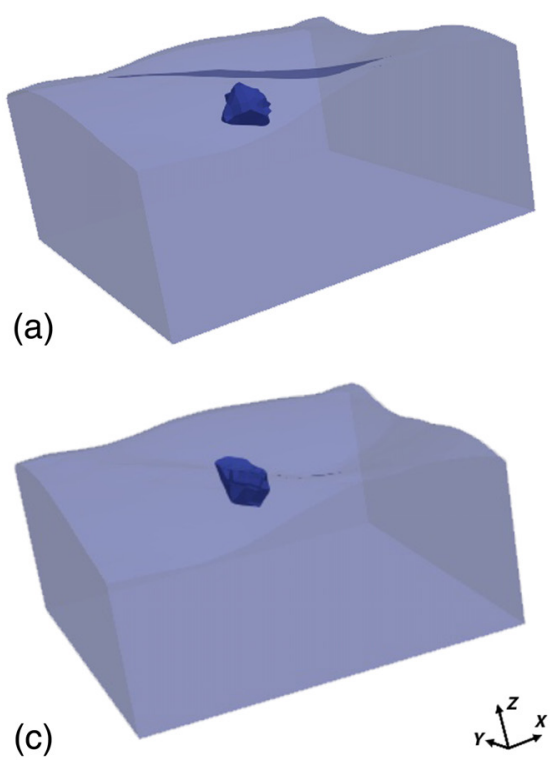

(b)
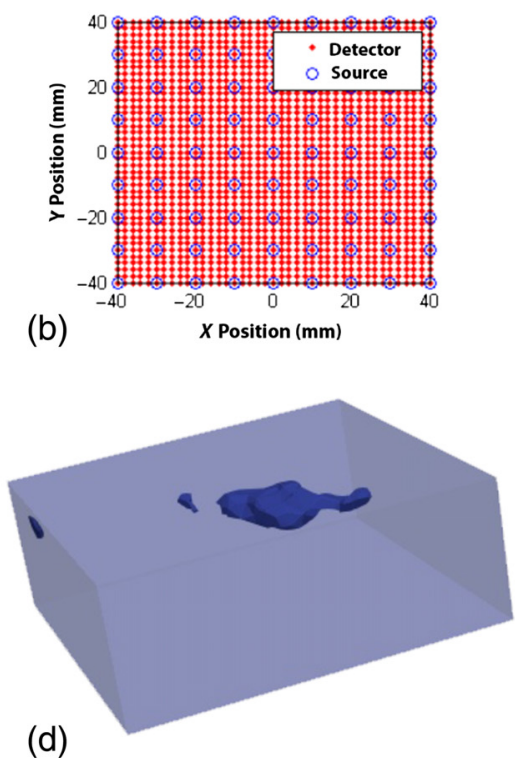

Fig. 4 The computer simulation results to demonstrate the influence of tissue geometry on 3-D reconstruction of blood flow distribution. (a) The assigned anomaly with a high-flow contrast of twofolds inside a breast-shaped volumetric mesh. (b) The source and detector locations in the $X-Y$ plane used for image reconstruction. (c) The reconstructed anomaly with a priori knowledge of the realistic surface geometry. (d) The reconstructed anomaly assuming a slab tissue volume with a flat surface. (a) were saved as a visualization toolkit format and ParaView (Kitware) was used for visualization, shown in the purple box of Fig. 3.

\subsection{Computer Simulations to Evaluate the Influence of Surface Geometry in Flow Reconstruction}

Computer simulations were conducted to evaluate the influence of irregular surface geometry on the reconstructed flow distributions (Fig. 4). The surface geometry used for the simulations was obtained by the PST from the breast of a patient immediately after mastectomy [Fig. 2(b)]. A solid volume mesh with the bottom area of $100 \times 100 \mathrm{~mm}^{2}$ and varied heights (depending on the breast surface geometry) was generated. The node distance was $1 \mathrm{~mm}$. The $9 \times 9$ sources and $41 \times 41$ detectors were distributed evenly to cover an ROI of $80 \times 80 \mathrm{~mm}^{2}$, which is identical to our realistic measurement setup for this patient. The background BFI was set as $10^{-8} \mathrm{~cm}^{2} / \mathrm{s}$, and a spherical anomaly (radius $=5 \mathrm{~mm}$ ) with a high-flow contrast of twofolds was created at the location of $(0,0,27)$ to match the forearm-cuff experiment result (Fig. 5). The reconstructions were performed with the realistic breast geometry and a flat surface of a slab $\left(100 \times 100 \times 30 \mathrm{~mm}^{3}\right)$, respectively, to evaluate the influence of surface geometry on the recovery of the anomaly flow contrast.

\subsection{3-D Imaging of Forearm Blood Flow Distributions During Arterial Cuff Occlusion}

The modified scDCT system integrating with the PST was first tested for continuous imaging of forearm blood flow responses to a cuff-occlusion on the upper arm (Fig. 5). This study was approved by the University of Kentucky Institutional Review Board (IRB). A healthy adult was asked to sit on a chair and extend her right arm on the table. An arterial cuff-occlusion with a pressure of $220 \mathrm{~mm} \mathrm{Hg}$ was applied for $\sim 160 \mathrm{~s}$ on the subject's upper right arm to induce blood flow changes 


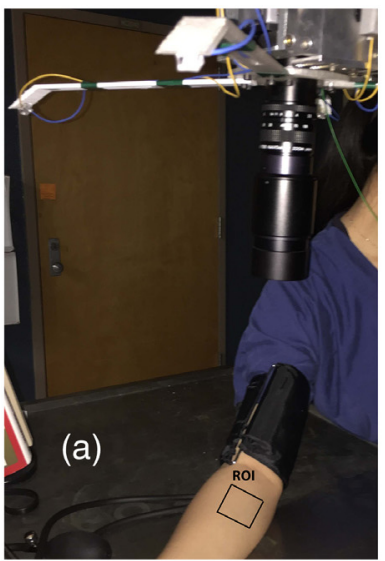

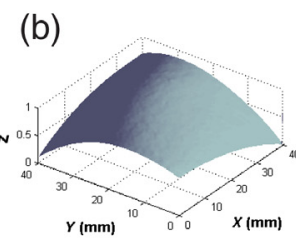

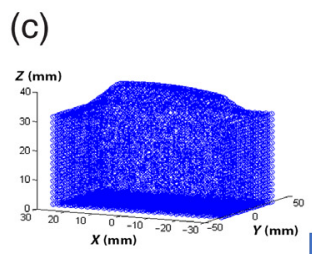

(d)

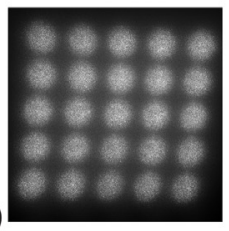

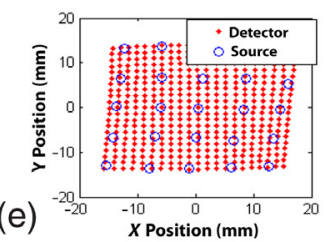

(e)

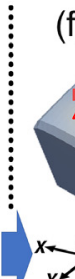

$(f): T 1$
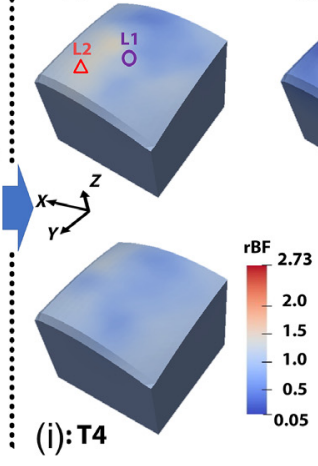

(g): T2

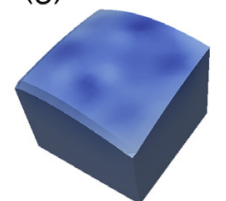

(h): T3
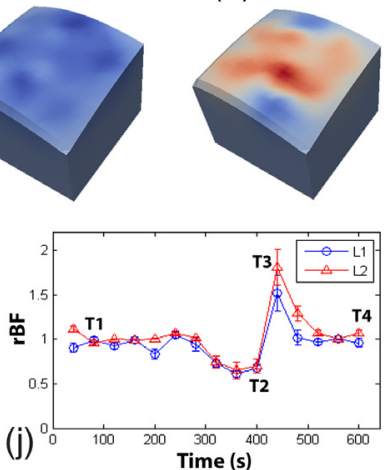

Fig. 5 3-D imaging of forearm blood flow distributions during arterial cuff occlusion. (a) scDCT measurement setup; (b) reconstructed forearm surface geometry with PST; (c) the tetrahedral mesh based on the forearm surface geometry; (d) the source distribution on the forearm surface; (e) the source and detector distribution on the $X-Y$ plane; (f)-(i) reconstructed 3-D distributions of forearm blood flow before (T1), during (T2), immediately after (T3), and in the recovery period (T4); and (j) time-course changes in rBF at the two locations (L1: 0, 0, 27 and L2: 12, 12, 33) throughout the cuff occlusion protocol.

in the forearm. The noncontact scDCT system was adjusted above the forearm to cover an ROI of $40 \times 40 \mathrm{~mm}^{2}$ on the forearm. The surface geometry was first obtained by the PST, followed by a sequential scanning of $5 \times 5$ sources on the ROI. Two frames at each source were taken and averaged to improve the SNR. The scDCT data were continuously acquired before, during, and after arterial cuff occlusion at the sampling rate of $0.025 \mathrm{~Hz}$. $21 \times 21$ detectors were distributed between the sources inside the ROI. The boundary BFIs were normalized to their baseline before occlusion to calculate the relative blood flow (rBF). A subject-specific solid volume mesh with node distance of $2 \mathrm{~mm}$ and bottom area of $60 \times 60 \mathrm{~mm}^{2}$ was generated for flow image reconstruction.

\subsection{Intraoperative Imaging of Mastectomy Skin Flaps}

The scDCT instrument was then moved to the surgical room for intraoperative imaging of mastectomy skin flaps. This study was approved by the IRB at the University of Kentucky. Two female subjects (ages: 64 and 48) undergoing single-stage mastectomy (direct-to-implant reconstruction) participated in this study. For the first patient (P1), after the incision was temporally closed using staples, the blood flow image was taken by the scDCT immediately after the nipple sparing mastectomy. For the second patient (P2), immediately after a skin sparing mastectomy and immediate reconstruction with an implant, fluorescence angiography (SPY Elite, Novadaq) ${ }^{27}$ with the injection of indocyanine green (ICG) dye was first used to determine the areas with low blood perfusion. The scDCT was then aligned to image a marked area with the lowest perfusion. An ROI of $80 \times 80 \mathrm{~cm}^{2}$ was selected for both patients, which is sufficient to cover the incision/suspension area. After getting the surface geometry by the PST, $9 \times 9$ sources were scanned over the ROI. Two frames at each source location were taken and averaged to improve the SNR. The total sampling time for the scDCT measurement was $\sim 2$ min. The $41 \times 41$ detectors were then distributed between the sources and inside the ROI for calculating boundary $\mathrm{rBF}$ data. A subject-specific solid volume mesh with the node distance of $2 \mathrm{~mm}$ and bottom area of $100 \times 100 \mathrm{~mm}^{2}$ was generated for flow image reconstruction.

\section{Results}

\subsection{Tissue Surface Geometry Affects Flow Image Reconstruction}

Figure 4 shows 3-D reconstruction results of blood flow distributions from computer simulations. Figure 4(a) shows the original assigned anomaly inside the breast-shaped volumetric mesh. Figure 4(b) shows the source and detector locations on the $X-Y$ plane. The reconstructed anomaly was segmented with the full width at half maximum criterion ${ }^{28}$ after normalizing to the background. The location and volume of the anomaly with $a$ priori knowledge of realistic surface geometry were recovered accurately [Fig. 4(c)]. The recovered peak blood flow of the anomaly was 1.8 -fold higher than the surrounding tissues, which agreed well with the assigned flow contrast (i.e., twofold, $8.5 \%$ error). The radius of the reconstructed anomaly was $\sim 5.7 \mathrm{~mm}$, which agreed fairly with the assigned value (i.e., $5 \mathrm{~mm}, \sim 14 \%$ error). The central location of the anomaly in the reconstructed flow was $(-0.8,-0.4,31.4)$, which also matched fairly the assigned location $(0.0,0.0,27.0)$. By contrast, large errors were observed when assuming a slab tissue volume with the flat surface [Fig. 4(c)]. The error in anomaly peak flow was $127 \%$. The anomaly shape was severely distorted, making it difficult to evaluate its size and location.

\subsection{Forearm Blood Flow Responses to Arterial Cuff Occlusion}

Figure 5 shows the measurement setup [Fig. 5(a)], data process [Figs. 5(b)-5(e)], and reconstructed results [Figs. 5(f)-5(j)] of rBF distributions in the forearm during arterial cuff occlusion. Figure 5(b) shows the tissue surface geometry of forearm (ROI: $40 \times 40 \mathrm{~mm}^{2}$ ) measured via PST. Figure 5(c) shows the volumetric tetrahedral mesh based on the obtained surface geometry. Figure 5(d) shows the source locations on the raw images and Fig. $5(\mathrm{e})$ shows distributions of sources $(5 \times 5)$ and detectors $(21 \times 21)$ inside the ROI. Figures 5(f)-5(i) show 3-D distributions of rBF changes in the forearm before (T1), during (T2), immediately after occlusion (T3), and in the recovery period (T4). Spatially heterogeneous responses in rBF distributions 
were observed over time in the imaged tissue volume at the ROI with the depth of around $10 \mathrm{~mm}$ (mean \pm standard deviation: T1: $0.95 \pm 0.14 ; \mathrm{T} 2: 0.545 \pm 0.16$; T3: $1.63 \pm 0.46$; and T4: $0.96 \pm 0.16)$. Figure $5(\mathrm{j})$ shows time course rBF changes inside two cubic volumes of $\sim 6 \mathrm{~mm}^{3}$ at the central locations of L1 $(0,0,27)$ and $\mathrm{L} 2(12,12,33)$, respectively. T1 to T4 represent the four timepoints corresponding to Figs. 5(f)-5(j). Global ischemia was observed during arterial occlusion due to the blockage of blood flow [Fig. 5(g)]. Reactive hyperemia was found immediately after releasing the cuff [Fig. 5(h)], followed by a flow recovery toward its preocclusion baseline [Fig. 5(i)]. These blood flow changes agreed with the expectation of forearm physiological changes due to the upper arm occlusion. ${ }^{16,29}$

\subsection{Blood Flow Distributions in Mastectomy Skin Flaps}

Figure 6 shows the scDCT imaging setup [Fig. 6(a)] and data processing procedures [Figs. 6(b)-6(e)] for patient \#1 (P1). Figure 6(b) shows the breast surface geometry (ROI: $80 \times 80 \mathrm{~mm}^{2}$ ) obtained by PST. Figure 6(c) shows the volumetric tetrahedral mesh based on the obtained surface geometry. Figure 6(d) shows the source locations on the raw images and Fig. 6(e) shows distributions of sources $(9 \times 9)$ and detectors $(41 \times 41)$ inside the ROI. Figure $6(\mathrm{f})$ shows the reconstructed 3-D image of $\mathrm{rBF}$ distribution in the skin flap immediately after mastectomy. Figure 6(g) shows the top view of 2-D cross section of $\mathrm{rBF}$ distributions at different depths of $0,3,6$, and $9 \mathrm{~mm}$. Spatial heterogeneity was observed in the imaged tissue volume (mean \pm standard deviation: $0.96 \pm 0.07)$.

Figure 7(a) shows the scDCT imaging setup for patient \#2 (P2). Figure 7(b) shows the reconstructed 3-D image of rBF reconstruction in the skin flap immediately after mastectomy and reconstruction. Figure 7(c) shows the top view of 2-D cross section of rBF distributions at different depths of 0,3 , 6 , and $9 \mathrm{~mm}$. Spatial heterogeneity was observed in the imaged tissue volume (mean \pm standard deviation: $0.98 \pm 0.49$ ). Figure 7(d) shows the 2-D fluorescence image of tissue perfusion in the skin flap. Interestingly, similar flow distribution patterns were observed from the top view of 3-D blood flow image $(\mathrm{scDCT})$ and the 2-D fluorescence perfusion image (SPY Elite, Novadaq).

\section{Discussion and Conclusions}

We have previously developed a noncontact scDCT system for 3-D imaging of blood flow distributions in relatively deep tissue

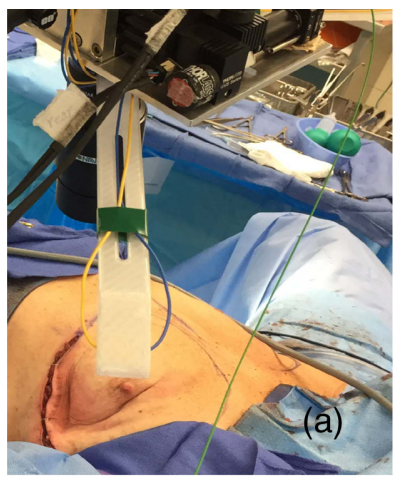

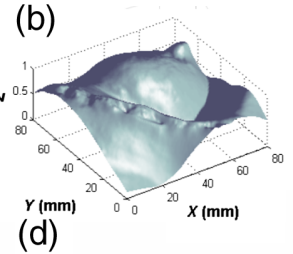
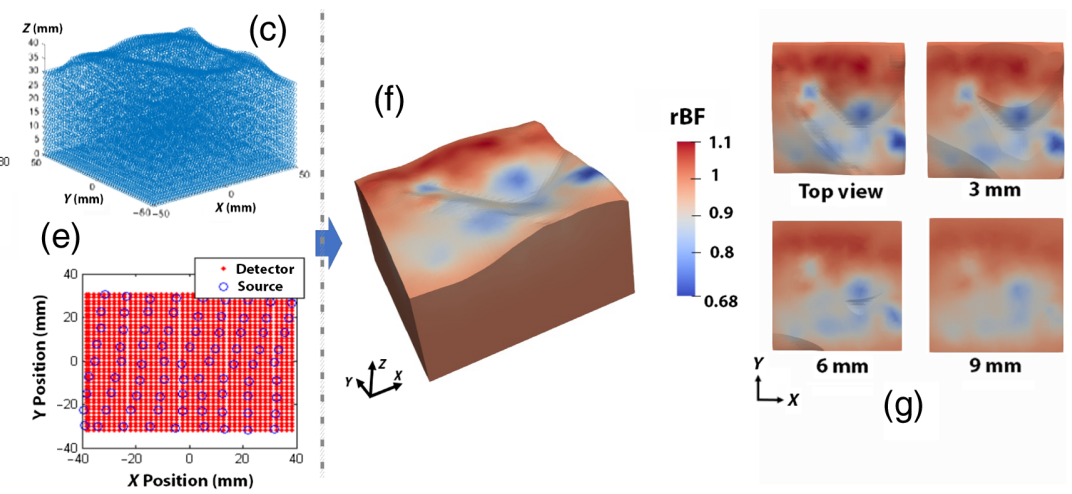

(d)

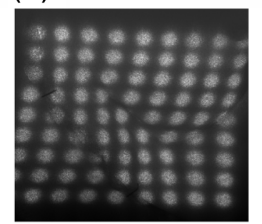

Fig. 6 Intraoperative 3-D imaging of blood flow distribution in a mastectomy skin flap (patient \#1) using the upgraded scDCT. (a) Imaging setup, (b) reconstructed tissue surface geometry with PST, (c) tetrahedral mesh-based on the surface geometry, (d) the source distribution on tissue surface, (e) the source and detector distribution on the $X-Y$ plane, (f) reconstructed 3-D blood flow distribution in mastectomy skin flap, and (g) 2-D cross-section views of flow distributions at different depths $(0,3,6$, and $9 \mathrm{~mm})$.
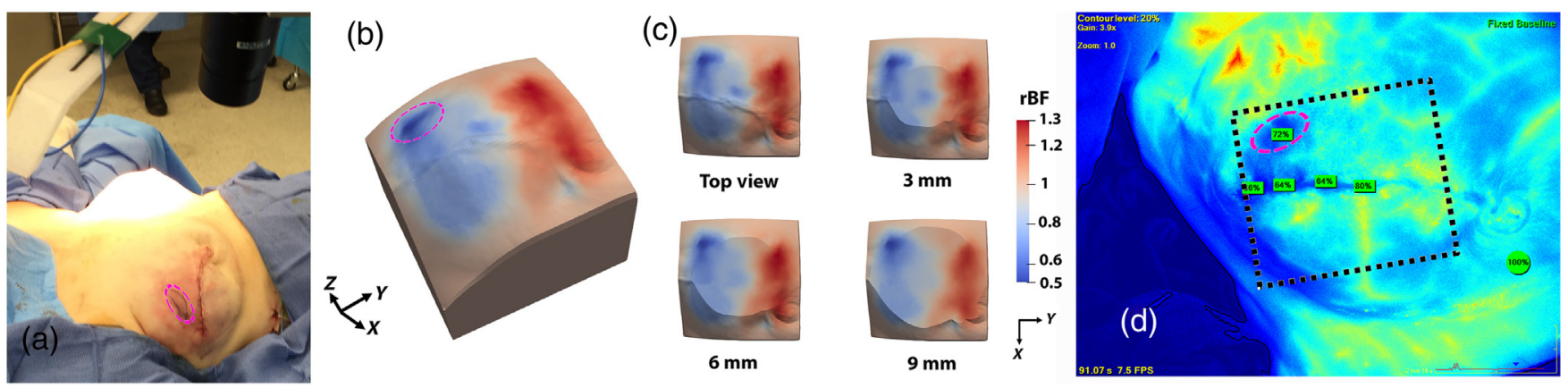

Fig. 7 Intraoperative imaging of blood flow distribution in a mastectomy skin flap (patient \#2) using the scDCT and SPY. (a) Imaging setup, (b) Reconstructed 3-D distribution of blood flow (scDCT), (c) 2-D cross-section views of flow distributions at different depths (scDCT), and (d) top view of blood perfusion measured by fluorescence angiography (SPY) with the injection of ICG. The dashed black box on the fluorescence image indicates the ROI for ScDCT imaging. The dashed red ellipses indicate the area with low blood perfusion observed from the SPY machine. 
volumes. ${ }^{16}$ The scDCT system provides many unique advanced features including fully noncontact hardware for avoiding tissue compression/interactions, rapid data acquisition through the diffuse speckle contrast method, adjustable S-D patterns/density for high-density measurements over a flexible FOV/ROI, FEM-based reconstruction algorithm, and portable and costefficient instrument design. Although the FEM-based method works with simple geometry as well as complex surface geometries, 3-D surface geometry has not been obtained for the image reconstruction. Instead, a semi-infinite tissue with a flat surface was assumed, which might affect the image reconstruction accuracy for tissues with irregular geometries.

This paper reports an upgraded scDCT system [Fig. 2(a)] that uses one single-CCD camera to obtain both tissue surface geometry (via PST) and boundary blood flow data (via scDCT). This innovative design allows us not only to leverage the advancements made previously from our scDCT prototype implementation but also to include surface geometry for improving image reconstruction. Furthermore, data acquisition times for both PST $(<1 \mathrm{~s})$ and scDCT (seconds to minutes depending on the selected ROI) are relatively short, which is important for clinical applications. Importantly, the integrated design with one single-CCD camera for both PST and scDCT data collection obviates the need for offline alignment of sources and detectors on the tissue boundary, thus offering the potential for online image reconstruction and display. This online processing ability is essential for intraoperative applications as it can provide real-time feedback to surgeons during surgery for optimizing the surgical procedure and outcome.

The new circuit was built to drive the LEDs and the mechanical arms were constructed using a 3-D printer to hold the LEDs. The PST and scDCT were innovatively integrated to form an upgraded scDCT [Fig. 2(a)], which enabled obtaining both tissue surface geometry (via PST) and boundary blood flow data (via scDCT). To run the upgraded scDCT system, several software packages were developed to operate the instrument (PST and scDCT) for data collection alternatively by a custom-made program (LabVIEW), and to perform data analysis and image reconstruction (MATLAB). After numerous tests, these software packages were optimized and standardized, as shown in Fig. 3.

Another improvement in this study is that the source locations were determined by extracting the pixels with regional high intensities on raw intensity images obtained by scDCT. In our previous studies with scDCT, the source locations were assumed evenly distributed over a selected ROI. ${ }^{16}$ This assumption can lead to large errors especially when imaging the tissues with irregular geometries, as shown in Figs. 5(d) and $6(d)$.

To evaluate the geometry influence on the image reconstruction, computer simulations were performed. Computer simulation is extensively used as a substitute economical tool for approval of the concept and for the optimization of performance. Results suggest that a priori knowledge of tissue surface geometry is crucial for precisely reconstructing the anomaly with blood flow contrast (Fig. 4). Image reconstruction with a volumetric mesh based on realistic tissue surface geometry recovers the anomaly blood flow contrast precisely. This improvement is essential for intraoperative imaging of mastectomy skin flaps as the flap surface geometries can vary greatly before and after the surgery.

The upgraded scDCT system was then tested for in vivo monitoring of human forearm blood flow responses to arterial cuff occlusion. This protocol has been extensively used for the validation of NIR instruments. ${ }^{16,29,30}$ The forearm has sufficient space to image, and the cuff occlusion can be easily applied on the upper arm to create blood flow variations. Both PST and scDCT data were collected by the upgraded scDCT system. Typical time-course ischemic and hyperemic responses during and after cuff occlusion are clearly observed at multiple locations of the forearm (Fig. 5). The spatial variation in blood flow response is expected because of regional hemodynamic response differences in the individual subject. Although $\mathrm{rBF}$ changes in the skin and muscle at different depths are similar in this subject [Fig. 5(j)], more subjects are needed to investigate potential differences in $\mathrm{rBF}$ responses between different types of tissues.

Finally, the upgraded scDCT was moved to the clinic for intraoperative imaging of mastectomy skin flaps. Through imaging two patients during surgery, we verified the feasibility and safety of our new system for intraoperative use. The total time for PST and scDCT measurements was $<2 \mathrm{~min}$, which is acceptable for clinical monitoring. We observed the expected spatial heterogeneities of blood flow distributions in the measured tissue volumes for both patients (Figs. 6 and 7). In the second patient, we got the chance to compare our 3-D blood flow image by the scDCT with the 2-D map of tissue perfusion by a commercial fluorescence angiography system (Fig. 7).

Fluorescence angiography is invasive (requiring dye injection), time-dependent, and limited to 2-D imaging of tissue perfusion. ${ }^{27}$ The 2-D fluorescence image represents an overlap of tissue perfusions over a depth of several millimeters. By contrast, our scDCT is noninvasive, time-independent, and has the ability to assess tissue blood flow distributions in three dimensions over a depth of $\sim 9 \mathrm{~mm}$. Since the average postoperative thickness of mastectomy skin flaps is $\sim 10 \mathrm{~mm},{ }^{31,32}$ our scDCT with a penetration depth of $\sim 9 \mathrm{~mm}$ is generally sufficient to detect skin flap ischemia/necrosis. Moreover, this maximal penetration depth is expected to be improved when using an advanced camera with better quality and sensitivity.

The general 2-D patterns of very low- and high-perfusion/ blood flow are similar in both florescence and scDCT images (Fig. 7). However, it is not surprising that there are discrepancies in small areas/regions between the two images due to the difference in imaging mechanism. The 3-D image of blood flow distribution over the entire tissue volume provides a more comprehensive assessment of skin flap ischemia/viability than the 2-D perfusion map on the tissue surface. For example, it may be used to assess/predict the partial-thickness or full-thickness skin flap necrosis. We expect this information can, after further studies in a large patient population, be used to provide intraoperative guidance of compromised tissue removal to prevent skin flap necrosis.

We would like to note a few limitations with the current scDCT system and study. Although effective, the FEM-based image reconstruction can take several hours depending on the sizes of mesh nodes and S-D pairs, which is not acceptable for real-time intraoperative monitoring. Recently, a fast and efficient sensitivity map generation in NIRFAST for diffuse optical tomography reconstruction has been proposed. This method utilizes a reduced sensitivity matrix that takes advantage of sparsity and parallelization processes, thus shortening the process time significantly, allowing for near real-time image recovery. ${ }^{33} \mathrm{We}$ 
will adapt this fast method to our modified NIRFAST for scDCT reconstruction in the future.

Although this study focuses on the 3-D imaging of blood flow distribution using the scDCT technique, we may explore a dual-wavelength scDCT system (i.e., adding another laser at a different wavelength) in the future, for simultaneous imaging of blood flow and oxygenation distributions to enhance the ability for assessing tissue viability.

In this study, we assumed the optical properties (absorption coefficient $\mu_{a}$ and reduced scattering coefficients $\mu_{s}^{\prime}$ ) from the literature. ${ }^{34}$ The boundary BFIs were normalized to the mean BFI value over the mastectomy (i.e., rBF) for image reconstruction. However, these parameters (i.e., $\mu_{a}, \mu_{s}^{\prime}$, and absolute BFI) may be quantified by taking the multiexposure and/or multiwavelength data at multiple S-D distances, ${ }^{35,36}$ which is the subject of our future work.

Finally, the sample size for each experimental protocol was small and none of the two patients had mastectomy skin flap necrosis. More patients are being measured to verify the capability of scDCT imaging for intraoperative prediction of mastectomy skin flap necrosis area/volume.

In conclusion, we have demonstrated the feasibility and safety of an upgraded scDCT system for 3-D imaging of blood flow distributions and variations in tissues with arbitrary geometries. The new scDCT uses one single-EMCCD camera to obtain, for the first time, both tissue surface geometry (via PST) and boundary blood flow data (via scDCT). This innovation not only leverages the advancements made previously from our scDCT prototype (i.e., noninvasive, noncontact, cost-effective, portable, flexible ROI/FOV, flexible sampling density, reflectance measurement, and 3-D imaging), but also includes the tissue surface geometry for improving image reconstruction accuracy. The in vivo imaging capability is demonstrated by imaging dynamic changes in forearm blood flow distribution during a cuff-occlusion procedure. The clinical viability is also evidenced by intraoperative imaging of mastectomy skin flaps and comparing with fluorescence angiography. The noncontact reflectance configuration with a flexible ROI and 3-D imaging capability make the scDCT applicable to multiple clinical applications including wounds, burns, trauma, and reconstructive tissue flaps.

\section{Disclosures}

G. Yu and C. Huang disclose the following patent owned by the University of Kentucky, which is related to the technology and analysis methods described in this study: G. Yu, Y. Lin, and C. Huang, "Noncontact three-dimensional diffuse optical imaging of deep tissue blood flow distribution," US Patent \#9861319 (2016 to 2036). This research was completed by co-authors unaffiliated with any commercial entity.

\section{Acknowledgments}

We acknowledge support from the National Institutes of Health Nos. R01-CA149274 (G. Y.), R21-AR062356 (G. Y.), and R21-HD091118 (G. Y.), American Heart Association Grant-In-Aid 16GRNT30820006 (G. Y.); National Endowment for Plastic Surgery, Plastic Surgery Foundation No. 3048112770 (L. W. and G. Y.); National Science Foundation EPSCoR \#1539068 (G. Y.); and the Halcomb Fellowship in Medicine and Engineering at the University of the Kentucky (S. M.).

\section{References}

1. G. Yu, "Diffuse correlation spectroscopy (DCS): a diagnostic tool for assessing tissue blood flow in vascular-related diseases and therapies," Curr. Med. Imaging Rev. 8(3), 194-210 (2012).

2. S. Fantini et al., "Cerebral blood flow and autoregulation: current measurement techniques and prospects for noninvasive optical methods," Neurophotonics 3(3), 031411 (2016).

3. D. A. Boas and A. K. Dunn, "Laser speckle contrast imaging in biomedical optics," J. Biomed. Opt. 15(1), 011109 (2010).

4. A. F. Fercher and J. D. Briers, "Flow visualization by means of single-exposure speckle photography," Opt. Commun. 37(5), 326330 (1981).

5. D. A. Boas and A. G. Yodh, "Spatially varying dynamical properties of turbid media probed with diffusing temporal light correlation," J. Opt. Soc. Am. A 14(1), 192-215 (1997).

6. D. A. Boas, L. E. Campbell, and A. G. Yodh, "Scattering and imaging with diffusing temporal field correlations," Phys. Rev. Lett. 75(9), 18551858 (1995)

7. C. Zhou et al., "Diffuse optical correlation tomography of cerebral blood flow during cortical spreading depression in rat brain," Opt. Express 14(3), 1125-1144 (2006).

8. A. Yodh and B. Chance, "Spectroscopy and imaging with diffusing light," Phys. Today 48(3), 34-40 (1995).

9. Y. Shang et al., "Portable optical tissue flow oximeter based on diffuse correlation spectroscopy," Opt. Lett. 34(22), 3556-3558 (2009).

10. C. Huang et al., "Noncontact diffuse optical assessment of blood flow changes in head and neck free tissue transfer flaps," J. Biomed. Opt. 20(7), 075008 (2015).

11. Y. Lin et al., "Three-dimensional flow contrast imaging of deep tissue using noncontact diffuse correlation tomography," Appl. Phys. Lett. 104(12), 121103 (2014)

12. C. Huang et al., "Alignment of sources and detectors on breast surface for noncontact diffuse correlation tomography of breast tumors," Appl. Opt. 54(29), 8808-8816 (2015).

13. H. Cheng et al., "Modified laser speckle imaging method with improved spatial resolution," J. Biomed. Opt. 8(3), 559-564 (2003).

14. S. Yuan et al., "Determination of optimal exposure time for imaging of blood flow changes with laser speckle contrast imaging," Appl. Opt. 44(10), 1823-1830 (2005).

15. N. B. Agochukwu et al., "A novel noncontact diffuse correlation spectroscopy device for assessing blood flow in mastectomy skin flaps: a prospective study in patients undergoing prosthesis-based reconstruction," Plast. Reconstr. Surg. 140(1), 26-31 (2017).

16. C. Huang et al., "Noncontact 3-D speckle contrast diffuse correlation tomography of tissue blood flow distribution," IEEE Trans. Med. Imaging 36, 2068-2076 (2017).

17. G. Yu, Y. Lin, and C. Huang, "Noncontact three-dimensional diffuse optical imaging of deep tissue blood flow distribution," US Letters Patent Application (15/07840) (2016).

18. C. Huang et al., "Speckle contrast diffuse correlation tomography of complex turbid medium flow," Med. Phys. 42(7), 4000-4006 (2015).

19. T. Li et al., "Simultaneous measurement of deep tissue blood flow and oxygenation using noncontact diffuse correlation spectroscopy flowoximeter," Sci. Rep. 3, 1358 (2013).

20. Y. Lin et al., "Noncontact diffuse correlation spectroscopy for noninvasive deep tissue blood flow measurement," J. Biomed. Opt. 17(1), 010502 (2012).

21. R. J. Woodham, "Photometric method for determining surface orientation from multiple images," Opt. Eng. 19(1), 191139 (1980).

22. Y. Zhang et al., "A fast 3-D reconstruction system with a low-cost camera accessory," Sci. Rep. 5, 10909 (2015).

23. C. H. Esteban, G. Vogiatzis, and R. Cipolla, "Multiview photometric stereo," IEEE Trans. Pattern Anal. Mach. Intell. 30(3), 548-554 (2008).

24. R. C. Gonzalez and R. E. Woods, "Morphological image processing," Chapter 9 in Digital Image Processing, Prentice Hall, Upper Saddle River, New Jersey (2002).

25. S. Yuan, "Sensitivity, noise and quantitative model of laser speckle contrast," PhD Thesis, Tufts University, Massachusetts (2008).

26. C. P. Valdes et al., "Speckle contrast optical spectroscopy, a non-invasive, diffuse optical method for measuring microvascular blood flow in tissue," Biomed. Opt. Express 5(8), 2769-2784 (2014). 
27. M. Griffiths, M. P. Chae, and W. M. Rozen, "Indocyanine green-based fluorescent angiography in breast reconstruction," Gland Surg. 5(2), 133-149 (2016)

28. H. Dehghani et al., "Three-dimensional optical tomography: resolution in small-object imaging," Appl. Opt. 42(16), 3117-3128 (2003).

29. M. Seong et al., "Simultaneous blood flow and blood oxygenation measurements using a combination of diffuse speckle contrast analysis and near-infrared spectroscopy," J. Biomed. Opt. 21(2), 027001 (2016).

30. C. Huang et al., "Low-cost compact diffuse speckle contrast flowmeter using small laser diode and bare charge-coupled-device," J. Biomed. Opt. 21(8), 080501 (2016).

31. J. D. Frey et al., "Mastectomy flap thickness and complications in nipple-sparing mastectomy: objective evaluation using magnetic resonance imaging," Plast. Reconstr. Surg. 5(8), e1439 (2017).

32. G. Pfeiler et al., "Residual breast tissue after mastectomy in non high risk and BRCA mutated patients," J. Clin. Oncol. 33(15 Suppl.), 10611061 (2015).

33. X. Wu et al., "Fast and efficient image reconstruction for high density diffuse optical imaging of the human brain," Biomed. Opt. Express 6(11), 4567-4584 (2015).

34. W. Mo et al., "Quantitative characterization of optical and physiological parameters in normal breasts using time-resolved spectroscopy: in vivo results of 19 Singapore women," J. Biomed. Opt. 14(6), 064004 (2009).

35. J. Liu et al., "Simultaneously extracting multiple parameters via multidistance and multi-exposure diffuse speckle contrast analysis," Biomed. Opt. Express 8(10), 4537-4550 (2017).

36. J. Liu et al., "Quantitative model of diffuse speckle contrast analysis for flow measurement," J. Biomed. Opt. 22(7), 076016 (2017).

Siavash Mazdeyasna is a PhD candidate in the Department of Biomedical Engineering at the University of Kentucky. He has been working on the development of speckle contrast diffusion correlation tomography (scDCT) for assessment of blood flow distribution in various tissues.

Chong Huang received his $\mathrm{PhD}$ in optical engineering in 2011. He is currently a research scientist in the Department of Biomedical
Engineering at the University of Kentucky. His research focuses on the development of diffuse optical technologies for noninvasive assessment of microvasculature hemodynamics in biological tissues.

Mingjun Zhao is a graduate student in the Department of Biomedical Engineering at the University of Kentucky. She has been working on the development of diffuse correlation spectroscopy and tomography for noninvasive assessment and imaging of blood flow distributions in animal and human tissues, including skeletal muscles.

Nneamaka B. Agochukwu received her MD degree from Vanderbilt University, Nashville, Tennessee, USA. She is currently a plastic surgery chief resident at the University of Kentucky. Her research and clinical interests include breast reconstruction and surgical outcomes.

Ahmed A. Bahrani received his MSc degree in medical engineering in 2006. He is now a PhD candidate in the Department of Biomedical Engineering at the University of Kentucky. He has been working on the development of the technology of quantification and analysis of cerebral blood flow using near-infrared diffuse correlation spectroscopy.

Lesley Wong is a professor in the Department of Surgery/Division of Plastic Surgery at the University of Kentucky. She has an active practice in breast reconstruction and general reconstruction as well as wound healing. She has collaborated with the Department of Biomedical Engineering to study the intraoperative assessment of perfusion in surgical tissues.

Guoqiang $\mathbf{Y u}$ is a professor of the Department of Biomedical Engineering at the University of Kentucky. He is the director of BioPhotonics Laboratory and leading a research group to develop various near-infrared diffuse spectroscopy and tomography systems for noninvasive imaging of deep tissue hemodynamics in animals and humans. 